International Journal of Pure and Applied Mathematics

Volume 114 No. $2 \quad 2017,203-219$

ISSN: 1311-8080 (printed version); ISSN: 1314-3395 (on-line version)

url: http://www.ijpam.eu

doi: 10.12732/ijpam.v114i2.4

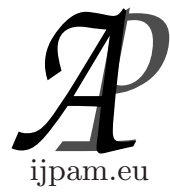

\title{
TOPOLOGICAL STRUCTURES OF \\ TYPE 2 FUZZY MULTISETS
}

\author{
Jaya Paul ${ }^{1} \S$, Sunil Jacob John ${ }^{2}$ \\ ${ }^{1,2}$ Department of Mathematics \\ National Institute of Technology \\ Calicut, INDIA
}

\begin{abstract}
In this paper the concept of topological spaces are extended in type 2 fuzzy multiset context. The structural properties such as open sets, closed sets, interior, closure and neighbourhoods in topological spaces are extended to type 2 fuzzy multi topological spaces and many related theorems are proved. In addition, the notions of base, sub-base and subspace of Type 2 fuzzy multi topology are discussed and properties studied. The paper concludes with the generalization of continuity in type 2 fuzzy multi topological spaces.
\end{abstract}

AMS Subject Classification: 54A40, 03E72

Key Words: type 2 fuzzy multisets, type 2 fuzzy multi topology, open sets, closed sets, interior, closure, base, sub-base, subspace

\section{Introduction}

In real world modelling applications, classical methods are insufficient to solve the complexities of uncertain data. To overcome the intrinsic vagueness and uncertainty involved in the parameters and variables in the models, many mathematical tools have arisen over the years such as fuzzy set theory, intuitionistic fuzzy set theory, soft set theory, rough set theory, vague set theory, interval mathematics etc., see $[1,6,12,15,21,22]$. Among these mathematical tools,

\footnotetext{
Received: $\quad$ October 9, 2016

Revised: $\quad$ February 20, 2017

Published: $\quad$ May 3, 2017

(c) 2017 Academic Publications, Ltd. url: www.acadpubl.eu

${ }^{\S}$ Correspondence author
} 
fuzzy set theory proposed by L. Zadeh in 1965 was the most popular one. It provides an appropriate frame work by allowing partial membership for representing the uncertain concepts which are imprecise or vague in nature. As an extension of ordinary fuzzy set Zadeh introduced the concept of type 2 fuzzy set which provides an additional degree of freedom that make possible to model the uncertainty in data directly. As compared to ordinary fuzzy sets, type 2 fuzzy sets are relatively hard to understand and clarify. But type 2 fuzzy set has the potential to outperform using ordinary fuzzy sets, especially in uncertain environments and can better improve a certain kind of interface with increasing imprecision and fuzziness in information. Operations on type- 2 fuzzy sets were studied by $[10,11]$.

In classical set theory, a set is a well defined collection of objects with non repetition of elements. But the situation in real life and science is not like this. In the practical problems there are enormous repetitions. To handle the situations with repetitions, one can use multi sets, a mathematical structure introduced by Cerf et al in 1972.[3] As a generalisation of multisets, Yager introduced fuzzy multisets and proposed possible applications to numerous decision making problems. In 1983, Atanassov introduced the concept of intuitionistic fuzzy set characterized by two functions expressing the degree of membership and the degree of nonmembership of elements of the universe.[2] Later the concept of intuitionistic fuzzy multiset is introduced in $[18,19]$ which has applications in medical diagnosis and robotics. In [9], the concept of type 2 fuzzy set theory is extended by combining type 2 fuzzy set and multiset, from which we can obtain a new mathematical model: Type 2 fuzzy multisets with improved performance for applications which require handling high levels of uncertainty.

In mathematics, topology can be formally defined as the study of properties with an associated family of subsets called topological spaces that are preserved under continuous maps. [14, 20] After introducing the notion of fuzzy sets and fuzzy set operations, several attempts have been made to develop mathematical structures using fuzzy set theory. In 1968, Chang[4] introduced fuzzy topology which provides a natural framework for generalizing many of the concepts of general topology to fuzzy topological spaces and its developments can be found in $[5,7,8,13,16,17]$. In this paper topologies on type 2 fuzzy multisets are provided and they can be useful for measuring the similarities and dissimilarities between the universes of objects with a multi nature in the secondary membership grades.

The paper is organized as follows. Section 2 is the preliminary section which recalls definitions and operations together with some properties of type 2 fuzzy multisets. In Section 3, we introduce the definitions of type 2 fuzzy multi 
topology and some of its structural properties such as type 2 fuzzy multi open sets, type 2 fuzzy multi closed sets, type 2 fuzzy multi interior, type 2 fuzzy multi closure and neighbourhood of a type 2 fuzzy multiset are studied. In Section 4 , base, sub-base, subspace for type 2 fuzzy multi topology are introduced and related theorems are also investigated. In Section 5, we generalize the notion of continuity to type 2 fuzzy multi context. In the final section, some concluding comments are presented.

\section{Preliminaries}

In this section, we will review some of the basic concepts and definitions which will be used in the sequel. For details, we refer [9].

\subsection{Type 2 Fuzzy Multisets [9]}

Let $X$ be a non empty, finite set called universe. A Type 2 fuzzy multiset denoted by $\tilde{A}$ drawn from $X$ is characterized by two membership functions.

Let $u_{i}$ denotes the primary membership grade and $\mu_{\tilde{A}}\left(x, u_{i}\right)$ denotes the secondary membership grade corresponding to $u_{i}$. Then $u_{i}$ is a mapping from $X$ to $I$ and $\mu_{\tilde{A}}\left(x, u_{i}\right)$ is a mapping from $X \times I$ to $M(I)$ where $I=[0,1]$ and $M(I)$ denotes the collection of crisp multisets drawn from $[0,1]$. That is $u_{i}: X \rightarrow I$ and $\mu_{\tilde{A}}\left(x, u_{i}\right): X \times I \rightarrow M(I)$.

A type 2 fuzzy multiset $\tilde{A}$ can be represented as

$$
\tilde{A}=\left\{\left(\left(x, u_{i}\right), \sum \mu_{\tilde{A}}\left(x, u_{i}\right) / x \in X, u_{i} \in J_{x} \subseteq I\right\},\right.
$$

where $0 \leq \mu_{\tilde{A}}\left(x, u_{i}\right) \leq 1$. Here $\sum$ indicates the multi nature of the secondary membership grades.

$\tilde{A}$ can also be expressed as

$$
\tilde{A}=\int_{x \in X} \int_{u_{i} \in J_{x}} \frac{\sum \mu_{\tilde{A}}\left(x, u_{i}\right)}{\left(x, u_{i}\right)}=\int_{x \in X} \frac{\left[\int_{u_{i} \in J_{x}} \sum f_{x}\left(u_{i}\right) / u_{i}\right]}{x},
$$

where $f_{x}\left(u_{i}\right)=\mu_{\tilde{A}}\left(x, u_{i}\right)$. Also the membership function of each $x \in X$ is denoted by $\tilde{A}(x)$. So $\tilde{A}(x)=\left[\int_{u_{i} \in J_{x}} \sum f_{x}\left(u_{i}\right) / u_{i}\right]$.

The class of all type 2 fuzzy multisets of the universe $X$ such that there is no limit on the multiplicity of the secondary membership grades is denoted by $\left[F M_{T 2}(X)\right]^{\infty}$.

The class of all type 2 fuzzy multisets of the universe $X$ with finite multiplicity is denoted by $F M_{T 2}(X)$. 
Now consider some special types of type 2 fuzzy multisets.

Definition 1. (Universal Type 2 Fuzzy Multisets, see [9]) If all the primary as well as secondary membership grades are unity then $\tilde{A}$ is known as Universal type 2 fuzzy multisets. That is, If all $\mu_{\tilde{A}}\left(x, u_{i}\right)=1$ and all $u_{i}=1$ then $\tilde{A}$ is called Universal type 2 fuzzy multiset. It is denoted by $\tilde{X}$.

Definition 2. (Null Type 2 Fuzzy Multisets, see [9]) If $\mu_{\tilde{A}}\left(x, u_{i}\right)=0$ for $u_{i} \neq 0 \neq 1$ for $u_{i}=0$ then $\tilde{A}$ is called Null type 2 fuzzy multiset. It is denoted by $\tilde{\phi}$.

\subsection{Basic Relations and Operations on Type 2 Fuzzy Multisets [9]}

In order to define the basic relations and operations on type 2 fuzzy multisets we need to arrange the membership functions in some order. So for each $x \in X$, let us first arrange the primary membership functions $u_{i}$ in a decreasingly ordered sequence. That is, $\left(u_{1}, u_{2}, u_{3}, u_{4} \ldots\right)$ such that $u_{1} \geq u_{2} \geq u_{3} \geq \ldots$.

Then for each $u_{i}$, arrange the secondary membership functions $\sum f_{x}\left(u_{i}\right)$ in a decreasingly ordered sequence. It is denoted by $\left(f_{x}^{1}\left(u_{i}\right), f_{x}^{2}\left(u_{i}\right), f_{x}^{3}\left(u_{i}\right), f_{x}^{4}\left(u_{i}\right), \ldots\right)$ such that $f_{x}^{1}\left(u_{i}\right) \geq f_{x}^{2}\left(u_{i}\right) \geq f_{x}^{3}\left(u_{i}\right) \geq f_{x}^{4}\left(u_{i}\right) \geq \ldots$

When we define an operation between two type 2 fuzzy multisets, say $\tilde{A}, \tilde{B}$, the length of both primary and secondary membership sequences should be set to be equal for each $x \in X$. In order to set the lengths of primary and secondary membership functions equal in $\tilde{A}$ and, $\tilde{B}$ we add sufficient number of zeroes at the end of the sequences.

Now let us discuss some basic relations and operations on type 2 fuzzy multisets. Let $\tilde{A}, \tilde{B}$ be two decreasingly ordered type 2 fuzzy multisets drawn from the same universe $X$ such that

$$
\begin{aligned}
& \tilde{A}=\int_{x \in X} \frac{\left[\int_{i=1}^{L_{p}(x)} \sum_{j=1}^{L_{s}(x)} f_{x}^{j}\left(u_{i}\right) / u_{i}\right]}{x}, \\
& \tilde{B}=\int_{x \in X} \frac{\left[\int_{i=1}^{L_{p}(x)} \sum_{j=1}^{L_{s}(x)} g_{x}^{j}\left(w_{i}\right) / w_{i}\right]}{x} .
\end{aligned}
$$

1. Inclusion: $\tilde{A}$ is a subtype 2 fuzzy multiset of $\tilde{B}$, denoted by $\tilde{A} \subseteq \tilde{B}$, if

$$
\begin{array}{rlrl}
u_{i} & \leq w_{i} & & \text { for } i=1,2,3, \ldots L_{p}(x) \text { and } \\
f_{x}^{j}\left(u_{i}\right) & \leq g_{x}^{j}\left(w_{i}\right) & \text { for } j=1,2,3, \ldots L_{s}(x), \text { for every } x \in X .
\end{array}
$$

2. Equality: $\tilde{A}$ and $\tilde{B}$ are equal, denoted by $\tilde{A}=\tilde{B}$, if

$u_{i}=w_{i}$ for $i=1,2,3, \ldots L_{p}(x)$ and

$f_{x}^{j}\left(u_{i}\right)=g_{x}^{j}\left(w_{i}\right)$, for $j=1,2,3, \ldots L_{s}(x)$, for every $x \in X$. 
3. Union: The union of $\tilde{A}$ and $\tilde{B}$, denoted by $\tilde{A} \cup \tilde{B}$, is given by

$$
\tilde{A} \cup \tilde{B}=\int_{x \in X} \int_{i=1}^{L_{p}(x)} \frac{\sum_{j=1}^{L_{s}(x)}\left[f_{x}^{j}\left(u_{i}\right) \star g_{x}^{j}\left(w_{i}\right)\right]}{u_{i} \vee v_{i}}
$$

4. Intersection: The intersection of $\tilde{A}$ and $\tilde{B}$, denoted by $\tilde{A} \cup \tilde{B}$, is defined by

$$
\tilde{A} \cap \tilde{B}=\int_{x \in X} \int_{i=1}^{L_{p}(x)} \frac{\sum_{j=1}^{L_{s}(x)}\left[f_{x}^{j}\left(u_{i}\right) \star g_{x}^{j}\left(w_{i}\right)\right]}{u_{i} \star v_{i}}
$$

5. Complement: The complement of $\tilde{A}$ is denoted by $\tilde{A}^{c}$

$$
\tilde{A}^{c}=\int_{x \in X} \frac{\left[\int_{u_{i} \in J_{x}} \sum f_{x}^{j}\left(u_{i}\right) /\left(1-u_{i}\right)\right]}{x} .
$$

where $\star$ represents a t-norm, $\vee$ represents the $\max \mathrm{t}$-conorm.

In addition to these operations, for $x, y \in X$ by $\tilde{A}(x) \geq \tilde{A}(y)$ it is taken that $u_{i} \geq w_{i}$ for $i=1,2,3, \ldots \max \left\{L_{p}(x), L_{p}(y)\right\}$ and $f_{x}^{j}\left(u_{i}\right) \geq f_{y}^{j}\left(w_{i}\right)$ for $j=1,2,3, \ldots \max \left\{L_{s}(x), L_{s}(y)\right\}$.

$\tilde{A}(x) \in \tilde{B}$ means $\tilde{B}(x) \geq \tilde{A}(x)$.

By $\tilde{A}(x) \vee \tilde{A}(y)$ means that $u_{i} \wedge w_{i}$ for $i=1,2,3, \ldots \max \left\{L_{p}(x), L_{p}(y)\right\}$ and $f_{x}^{j}\left(u_{i}\right) \vee f_{y}^{j}\left(w_{i}\right)$ for $j=1,2,3, \ldots \max \left\{L_{s}(x), L_{s}(y)\right\}$

By $\tilde{A}(x) \wedge \tilde{A}(y)$ means that $u_{i} \wedge w_{i}$ for $i=1,2,3, \ldots \max \left\{L_{p}(x), L_{p}(y)\right\}$ and $f_{x}^{j}\left(u_{i}\right) \wedge f_{y}^{j}\left(w_{i}\right)$ for $j=1,2,3, \ldots \max \left\{L_{s}(x), L_{s}(y)\right\}$.

\section{Operations under collection of type 2 fuzzy multisets}

Let $\left\{\tilde{A}_{\alpha}\right\}_{\alpha \in J}$ be an arbitrary collection of type 2 fuzzy multisets drawn from $X$. Then the following operations are possible under an arbitrary collection of type 2 fuzzy multisets.

(1) The union $\cup_{\alpha \in J} \tilde{A}_{\alpha}$ is defined as

$$
\left[\cup_{\alpha \in J} \tilde{A}_{\alpha}\right](x)=\vee_{\alpha \in J} \tilde{A}_{\alpha}(x)=\int_{i=1}^{L_{p}(x)} \frac{\sum_{j=1}^{L_{s}(x)} \vee_{\alpha \in J}\left[f_{x}^{j}\left(u_{i}\right)\right]_{\alpha}}{\vee_{\alpha \in J}\left[u_{i}\right]_{\alpha}}
$$

(2) The intersection $\cap_{\alpha \in J} \tilde{A}_{\alpha}$ is defined as

$$
\left[\cap_{\alpha \in J} \tilde{A}_{\alpha}\right](x)=\wedge_{\alpha \in J} \tilde{A}_{\alpha}(x)=\int_{i=1}^{L_{p}(x)} \frac{\sum_{j=1}^{L_{s}(x)} \wedge_{\alpha \in J}\left[f_{x}^{j}\left(u_{i}\right)\right]_{\alpha}}{\wedge_{\alpha \in J}\left[u_{i}\right]_{\alpha}}
$$

where $\vee$ and $\wedge$ represents the supremum and infimum values of the membership grades.

Remark : Every nonempty set of real numbers that have upper bound has 
a supremum and that have lower bound has an infimum. Thus, the arbitrary union and arbitrary intersection defined are closed under the collection $\left\{\tilde{A}_{\alpha}\right\}_{\alpha \in J}$, because the collection $\left\{\tilde{A}_{\alpha}\right\}_{\alpha \in J}$ drawn from $F M_{T 2}(X)$ contains elements with finite multiplicity in both primary and secondary membership grades for each $x \in X$.

Theorem 3. Let $\left\{\tilde{A}_{\alpha}\right\}_{\alpha \in J}$ be an arbitrary collection of type 2 fuzzy multisets drawn from $X$ and $\tilde{B}$ be another type 2 fuzzy multiset drawn from $X$. Then

(1) $\tilde{B} \wedge\left(\bigvee_{\alpha \in J} \tilde{A}_{\alpha}\right)=\bigvee_{\alpha \in J}\left(\tilde{B} \wedge \tilde{A}_{\alpha}\right)$

(2) $\tilde{B} \vee\left(\bigwedge_{\alpha \in J} \tilde{A}_{\alpha}\right)=\bigwedge_{\alpha \in J}\left(\tilde{B} \vee \tilde{A}_{\alpha}\right)$

(3) $1-\left(\bigvee_{\alpha \in J} \tilde{A}_{\alpha}\right)=\bigwedge_{\alpha \in J}\left(1-\tilde{A}_{\alpha}\right)$

(4) $1-\left(\bigwedge_{\alpha \in J} \tilde{A}_{\alpha}\right)=\bigvee_{\alpha \in J}\left(1-\tilde{A}_{\alpha}\right)$

\section{Type 2 Fuzzy Multi Topological Spaces}

In this section we introduced the concept of type 2 fuzzy multi topology.

Definition 4. Let $\tau$ be the collection of type 2 fuzzy multisets over $X$; then $\tau$ is said to be a type 2 fuzzy multi topology on $X$ if

(1) $\tilde{\phi}, \tilde{X}$ belong to $\tau$,

(2) the union of any number of type 2 fuzzy multisets in $\tau$ belongs to $\tau$,

(3) the intersection of any two type 2 fuzzy multisets in $\tau$ belongs to $\tau$. The pair $(X, \tau)$ is called a type 2 fuzzy multi topological space over $X$.

Definition 5. Let $(X, \tau)$ be a type 2 fuzzy multi topological space over $X$; then the members of $\tau$ are said to be type 2 fuzzy multi open sets in $X$.

Definition 6. Let $(X, \tau)$ be a type 2 fuzzy multi topological space over $X$. A type 2 fuzzy multiset $\tilde{A}$ is said to be a type 2 fuzzy multi closed set in $X$, if its complement $\tilde{A}^{c}$ belongs to $\tau$.

Theorem 7. Let $(X, \tau)$ be a type 2 fuzzy multi topological space. Then the following conditions hold:

(1) $\tilde{\phi}, \tilde{X}$ are type 2 fuzzy multi closed sets.

(2) Arbitrary intersection of type 2 fuzzy multi closed sets is a closed set.

(3) Finite union of type 2 fuzzy multi closed sets is a closed set.

Proof. (1) $\tilde{\phi}, \tilde{X}$ are type 2 fuzzy multi closed sets because they are the complements of the type 2 fuzzy multi open sets $\tilde{X}, \tilde{\phi}$ respectively.

(2) Let $\left\{\tilde{A}_{\alpha}\right\}_{\alpha \in J}$ be a collection of type 2 fuzzy multi closed sets. Then $\left[\cap_{\alpha \in J} \tilde{A}_{\alpha}\right](x)=\wedge_{\alpha \in J} \tilde{A}_{\alpha}(x)$ 


$$
\begin{aligned}
& =\int_{i=1}^{L_{p}(x)} \frac{\sum_{j=1}^{L_{s}(x)} \wedge_{\alpha \in J}\left[f_{x}^{j}\left(u_{i}\right)\right]_{\alpha}}{\wedge_{\alpha \in J}\left[u_{i}\right]_{\alpha}} \\
& =\int_{i=1}^{L_{p}(x)} \frac{\sum_{j=1}^{L_{s}(x)} \wedge_{\alpha \in J}\left[f_{x}^{j}\left(u_{i}\right)\right]_{\alpha}}{1-\left(\vee_{\alpha \in J}\left[1-u_{i}\right]\right)_{\alpha}} \quad \text { (by theorem 3) } \\
& =\left[\vee_{\alpha \in J} \tilde{A}_{\alpha}^{c}\right]^{c}(x) \\
& =\left[\cup_{\alpha \in J} \tilde{A}_{\alpha}^{c}\right]^{c}(x) .
\end{aligned}
$$

Since arbitrary union of type 2 fuzzy multi open sets are open $\left[\cup_{\alpha \in J} \tilde{A}_{\alpha}^{c}\right]$ is an open set and therefore $\left[\cap_{\alpha \in J} \tilde{A}_{\alpha}\right]$ is a type 2 fuzzy closed set.

(3) Similarly, if $\tilde{A}_{i}$ is closed, for $i=1,2, \ldots n$ then $\cup_{i=1}^{n} \tilde{A}_{i}$ is a type 2 fuzzy multi closed set. (Since finite intersections of type 2 fuzzy multi open sets is open).

Proposition 8. Let $\left\{\tau_{k} \mid k \in K\right\}$ be a family of all type 2 fuzzy multi topologies on $X$; then $\cap_{k \in K} \tau_{k}$ is a type 2 fuzzy multi topology on $X$.

Proof. (1) $\tilde{\phi}$ and $\tilde{X}$ belong to $\cap_{k \in K} \tau_{k}$.

(2) Let $\left\{\tilde{A}_{\alpha} \mid \alpha \in J\right\} \subseteq \cap_{k \in K} \tau_{k}$. Then for all $\alpha \in J, \tilde{A}_{\alpha} \in \tau_{k}$, so $\cup_{\alpha \in J} \tilde{A}_{\alpha} \in \tau_{k}$. Thus $\cup_{\alpha \in J} \tilde{A}_{\alpha} \in \cap_{k \in K} \tau_{k}$.

(3) Let $\tilde{A}, \tilde{B} \in \cap_{k \in K} \tau_{k}$. Then for all $k \in K, \tilde{A}, \tilde{B} \in \tau_{k}$. Since $\tau_{k}$ are all type 2 fuzzy multi topologies $\tilde{A} \cap \tilde{B} \in \tau_{k}$ for all $k \in K$, so $\tilde{A} \cap \tilde{B} \in \cap_{k \in K} \tau_{k}$.

Definition 9. Let $\left(X, \tau_{1}\right)$ and $\left(X, \tau_{2}\right)$ be two type 2 fuzzy multi topological spaces over the same universe $X$. If each type 2 fuzzy multiset $\tilde{A} \in \tau_{1}$ is in $\tau_{2}$, then $\tau_{2}$ is called type 2 fuzzy multi finer than $\tau_{1}$, or $\tau_{1}$ is type 2 fuzzy multi coarser than $\tau_{2}$.

Proposition 10. Let $\left\{\tau_{k} \mid k \in K\right\}$ be a family of all type 2 fuzzy multi topologies on $X$; then $\cap_{k \in K} \tau_{k}$ is the coarsest type 2 fuzzy multi topology on $X$.

Proof. $\cap_{k \in K} \tau_{k}$ is a type 2 fuzzy multi topology on $X$ by proposition 2.4. If each type 2 fuzzy multiset $\tilde{A} \in \cap_{k \in K} \tau_{k}$, then $\tilde{A} \in \tau_{k}$ for all $k \in K$. Hence $\cap_{k \in K} \tau_{k}$ is the coarsest type 2 fuzzy multi topology on $X$.

Remark : Let $\left(X, \tau_{1}\right)$ and $\left(X, \tau_{2}\right)$ be two type 2 fuzzy multi topological spaces over the same universe $X$; then $\left(X, \tau_{1} \cup \tau_{2}\right)$ (need not be a type 2 fuzzy multi topological space over $X$.

Example:Let $X=\left\{x_{1}, x_{2}\right\}$, and $\tau_{1}=\{\tilde{\phi}, \tilde{X}, \tilde{A}\}, \tau_{2}=\{\tilde{\phi}, \tilde{X}, \tilde{B}\}$ be two type 2 fuzzy multi topologies defined on $X$ where $\tilde{A}, \tilde{B}$ defined as follows:

$$
\begin{aligned}
\tilde{A}= & \frac{\{1,0.3\} / 0.8+\{0.7,0.5,0.5\} / 0.6+\{0.6,0.3,0.3\} / 0.3}{x_{1}}+ \\
& \frac{\{0.9,0.4\} / 0.8+\{1,1,0.4\} / 0.5+\{0.5,0.2\} / 0.4}{x_{2}}
\end{aligned}
$$




$$
\begin{aligned}
\tilde{B}= & \frac{\{0.8,0.3\} / 0.6+\{0.5,0.4,0.4\} / 0.5+\{0.5,0.4,0.2\} / 0.4}{x_{1}}+ \\
& \frac{\{0.8,0.5\} / 1+\{1,1,0.7\} / 0.7+\{0.4,0.2\} / 0.4}{x_{2}}
\end{aligned}
$$

Now $\tau_{1} \cup \tau_{2}=\{\tilde{\phi}, \tilde{X}, \tilde{A}, \tilde{B}\}$. If we take $\tilde{C}=\tilde{A} \cup \tilde{B}$ and $\tilde{D}=\tilde{A} \cap \tilde{B}$ then

$$
\begin{aligned}
\tilde{C}= & \frac{\{0.8,0.3\} / 0.8+\{0.5,0.4,0.4\} / 0.6+\{0.5,0.3,0.2\} / 0.4}{x_{1}}+ \\
& \frac{\{0.8,0.4\} / 1+\{1,1,0.4\} / 0.7+\{0.4,0.2\} / 0.4}{x_{2}} \\
\tilde{D}= & \frac{\{0.8,0.3\} / 0.6+\{0.5,0.4,0.4\} / 0.6+\{0.5,0.3,0.2\} / 0.3}{x_{1}}+ \\
& \frac{\{0.8,0.4\} / 0.8+\{1,1,0.4\} / 0.5+\{0.4,0.2\} / 0.4}{x_{2}}
\end{aligned}
$$

clearly, $\tilde{C} \notin \tau_{1} \cup \tau_{2}, \tilde{D} \notin \tau_{1} \cup \tau_{2}$, so $\left(X, \tau_{1} \cup \tau_{2}\right)$ is not a type 2 fuzzy multi topological space on $X$.

Definition 11. Let $(X, \tau)$ be a type 2 fuzzy multi topological space and let $\tilde{A}$ be a type 2 fuzzy multiset over $X$. Then the type 2 fuzzy multi interior of $\tilde{A}$, denoted by $\tilde{A}^{o}$, is defined as the union of all type 2 fuzzy multi open sets contained in $\tilde{A}$.

Clearly, $\tilde{A}^{o}$ is the largest type 2 fuzzy multi open set contained in $\tilde{A}$.

Theorem 12. Let $(X, \tau)$ be a type 2 fuzzy multi topological space over $X$, and let $\tilde{A}$ and $\tilde{B}$ be two type 2 fuzzy multisets over $X$. Then the following properties hold:

(1) $\tilde{\phi}^{o}=\tilde{\phi}$ and $\tilde{X}^{o}=\tilde{X}$

(2) $\tilde{A}^{o} \subseteq \tilde{A}$

(3) $\tilde{A}$ is a type 2 fuzzy multi open set if and only if $\tilde{A}^{o}=\tilde{A}$

(4) $\left(\tilde{A}^{o}\right)^{o}=\tilde{A}^{o}$

(5) $\tilde{A} \subseteq \tilde{B}$ implies $\tilde{A}^{o} \subseteq \tilde{B}^{o}$

(6) $\tilde{A}^{o} \cap \tilde{B}^{o}=(\tilde{A} \cap \tilde{B})^{o}$.

Proof. (1) and (2) are trivial.

(3) If $\tilde{A}$ is a type 2 fuzzy multi open set, then $\tilde{A} \subseteq \tilde{A}^{o}$. Since $\tilde{A}^{o} \subseteq \tilde{A}$ by part

(2), so $\tilde{A}^{o}=\tilde{A}$.

(4) Since $\tilde{A}^{o}$ is atype 2 fuzzy multi open set, we have $\left(\tilde{A}^{o}\right)^{o}=\tilde{A}^{o}$ by part $(3)$.

(5) If $\tilde{A} \subseteq \tilde{B}$, then $\tilde{A}^{o} \subseteq \tilde{A} \subseteq \tilde{B}$. $\tilde{A}^{o}$ is a type 2 fuzzy multi open set contained 
in $\tilde{B}$, so $\tilde{A}^{o} \subseteq \tilde{B}^{o}$ by definition (3.7).

(6) Since $(\tilde{A} \cap \tilde{B}) \subseteq \tilde{A},(\tilde{A} \cap \tilde{B}) \subseteq \tilde{B}$, so $(\tilde{A} \cap \tilde{B})^{o} \subseteq \tilde{A}^{o},(\tilde{A} \cap \tilde{B})^{o} \subseteq \tilde{B}^{o}$ by part (5). Thus $(\tilde{A} \cap \tilde{B})^{o} \subseteq \tilde{A}^{o} \cap \tilde{B}^{o}$. Since $\tilde{A}^{o} \cap \tilde{B}^{o} \subseteq \tilde{A} \cap \tilde{B}$, so $\left(\tilde{A}^{o} \cap \tilde{B}^{o}\right)^{o} \subseteq(\tilde{A} \cap \tilde{B})^{o}$ by part (5). $\tilde{A}^{o} \cap \tilde{B}^{o}$ is a type 2 fuzzy multi open set; then $\left(\tilde{A}^{o} \cap \tilde{B}^{o}\right)^{o}=(\tilde{A} \cap \tilde{B})^{o}$ by part $(3)$. Hence $\tilde{A}^{o} \cap \tilde{B}^{o}=(\tilde{A} \cap \tilde{B})^{o}$.

Definition 13. Let $(X, \tau)$ be a type 2 fuzzy multi topological space, and let $\tilde{A}$ be a type 2 fuzzy multiset over $X$. Then the type 2 fuzzy multi closure of $\tilde{A}$, denoted by $\operatorname{cl}(\tilde{A})$, is defined as the intersection of all type 2 fuzzy closed sets containing $\tilde{A}$.

Clearly, $\operatorname{cl}(\tilde{A})$ is the smallest type 2 fuzzy multi closed set containing $\tilde{A}$.

Theorem 14. Let $(X, \tau)$ be a type 2 fuzzy multi topological space over $X$, and let $\tilde{A}$ and $\tilde{B}$ be two type 2 fuzzy multisets over $X$. Then the following properties hold:

(1) $\operatorname{cl}(\tilde{\phi})=\tilde{\phi}$ and $\operatorname{cl}(\tilde{X})=\tilde{X}$

(2) $\tilde{A} \subseteq \operatorname{cl}(\tilde{A})$

(3) $\tilde{A}$ is a type 2 fuzzy multi closed set if and only if $\operatorname{cl}(\tilde{A})=\tilde{A}$

(4) $\operatorname{cl}(\operatorname{cl}(\tilde{A}))=\operatorname{cl}(\tilde{A})$

(5) $\tilde{A} \subseteq \tilde{B}$ implies $\operatorname{cl}(\tilde{A}) \subseteq \operatorname{cl}(\tilde{B})$

(6) $\operatorname{cl}(\tilde{\tilde{A}}) \cap \operatorname{cl}(\tilde{B})=\operatorname{cl}(\tilde{A} \cap \tilde{B})$.

Proof. The proof is similar to the proof of theorem (12).

Definition 15. Let $(X, \tau)$ be a type 2 fuzzy multi topological space and $\tilde{N} \in \tau$. Then $\tilde{N}$ is said to be a neighbourhood, or nbhd for short, of a type 2 fuzzy multiset $\tilde{A}$ if there exist a type 2 fuzzy multi open set $\tilde{U}$ such that $\tilde{A} \subseteq \tilde{U} \subseteq \tilde{N}$.

Theorem 16. A type 2 fuzzy multiset $\tilde{A}$ is open if and only if for each type 2 fuzzy multiset $\tilde{B}$ contained in $\tilde{A}, \tilde{A}$ is a nbhd of $\tilde{B}$.

Proof. Clearly, if $\tilde{A}$ is open and $\tilde{B} \subseteq \tilde{A}$ then $\tilde{A}$ is a nbhd of $\tilde{B}$.

Conversely, since $\tilde{A} \subseteq \tilde{A}$, there exists a type 2 fuzzy multi open set $\tilde{U}$ such that $\tilde{A} \subseteq \tilde{U} \subseteq \tilde{A}$. Hence, $\tilde{A}=\tilde{U}$ and $\tilde{A}$ is open.

Theorem 17. If $\aleph$ is the nbhd system of a type 2 fuzzy multiset $\tilde{A}$, then the finite intersections of members of $\aleph$ belongs to $\aleph$ and each type 2 fuzzy multiset which contains a member of $\aleph$ belongs to

Proof. Let $\tilde{N}_{1}, \tilde{N}_{2}$ be nbhds of a type 2 fuzzy multiset $\tilde{A}$. Then there exists open nbhds $\tilde{U}_{1}$ and $\tilde{U}_{2}$ contained in $\tilde{N}_{1}$ and $\tilde{N}_{2}$, respectively. Then $\tilde{N}_{1} \cap \tilde{N}_{2}$ contains the open nbhd $\tilde{U}_{1} \cap \tilde{U}_{2}$ and hence a nbhd of $\tilde{A}$. Thus the intersection of two (and hence of any finite number of) members of $\aleph$ is a member of $\aleph$. 
Hence, if a type 2 fuzzy multiset $\tilde{B}$ contains a nbhd of $\tilde{A}$ it contains an open nbhd of $\tilde{A}$ and consequently is itself a nbhd.

\section{Type 2 Fuzzy Multi Bases and Sub-Bases}

Definition 18. Let $(X, \tau)$ be a type 2 fuzzy topological space and $\vartheta$ be a subfamily of $\tau$. If every member of $\tau$ can be written as the type 2 fuzzy multi union of some members of $\vartheta$, then $\vartheta$ is called a type 2 fuzzy multi base for the type 2 fuzzy multi topology $\tau$.

Lemma 19. Let $(X, \tau)$ be a type 2 fuzzy topological space and $\vartheta$ be a type 2 fuzzy multi base for $\tau$. Then $\tau$ equals the collection of type 2 fuzzy multi unions of elements of $\vartheta$.

Lemma 20. Let $(X, \tau)$ and $\left(X, \tau_{1}\right)$ be two type 2 fuzzy topological spaces and $\vartheta$ and $\vartheta_{1}$ be type 2 fuzzy multi bases for $\tau$ and $\tau_{1}$ respectively. If $\vartheta \subset \vartheta_{1}$, then $\tau$ is type 2 fuzzy multi finer than $\tau_{1}$.

Lemma 21. Let $(X, \tau)$ be a type 2 fuzzy topological space and $\boldsymbol{B}$ is a collection of type 2 fuzzy multi open sets in $\tau$ such that for each $\tilde{U} \in \tau$, there is an element $\tilde{B} \in \boldsymbol{B}$ with $\tilde{B} \subseteq \tilde{U}$. Then $\boldsymbol{B}$ is a type 2 fuzzy multi base for $\tau$.

The next theorem gives a criterion for determining whether a type 2 fuzzy multi topology on $X$ is finer than another in terms of type 2 fuzzy multi bases.

Theorem 22. Let $\vartheta$ and $\vartheta^{\prime}$ are type 2 fuzzy multi bases for the topologies $\tau$ and $\tau^{\prime}$ respectively. Then the following are equivalent:

(1) $\tau^{\prime}$ is finer than $\tau$.

(2) For each $B \in \vartheta$, there is an element $B^{\prime} \in \vartheta^{\prime}$ such that $B^{\prime} \subseteq B$.

Proof. $(1) \Rightarrow(2)$. Let $B \in \vartheta$. Then $B \in \tau$ by definition and $\tau \subseteq \tau^{\prime}$ by (1). Therefore $B \in \vartheta^{\prime}$. Since $\tau^{\prime}$ is generated by $\vartheta^{\prime}$, there is a base element $B^{\prime} \in \vartheta^{\prime}$ such that $B^{\prime} \subseteq B$.

$(2) \Rightarrow(1)$. Given an element $\tilde{U}$ of $\tau$, we show that $\tilde{U} \in \tau^{\prime}$.

$\tilde{U} \in \tau \Rightarrow$ there is an element $B \in \vartheta$ such that $B \subseteq \tilde{U}$ (since $\vartheta$ generates $\tau$ ).

From (2) there is an element $B^{\prime} \in \vartheta^{\prime}$ such that $B^{\prime} \subseteq B$.

Then $B^{\prime} \subseteq \tilde{U}$ and hence $\tilde{U} \in \vartheta^{\prime}$.

Definition 23. Let $(X, \tau)$ and $(Y, \varsigma)$ be two type 2 fuzzy multi topological spaces. The type 2 fuzzy multi topological space $Y$ is called a subspace of the type 2 fuzzy multi topological space $X$ if $Y \subseteq X$ and the open subsets of $Y$ are precisely of the form $\tau_{\tilde{Y}}=\left\{\tilde{U}^{\prime}=\tilde{Y} \cap \tilde{U}: \tilde{U} \in \tau\right\}$.

Here we may say that each open subset $\tilde{U}^{\prime}$ of $Y$ is the restriction to $\tilde{Y}$ of an 
open subset $\tilde{U}$ of $X$.

That is, $(Y, \varsigma)$ is called a subspace of $(X, \tau)$ if the type 2 fuzzy multi open sets of $Y$ are the type 2 fuzzy multi intersections of open sets of $X$ with $\tilde{Y}$.

Theorem 24. Let $(X, \tau)$ be a type 2 fuzzy multi topological space. If $\vartheta$ is a type 2 fuzzy multi base for $\tau$ then the collection $\vartheta_{Y}=\{B \cap \tilde{Y}: B \in \vartheta\}$ is a type 2 fuzzy multi base for the type 2 fuzzy multi subspace topology on $Y \subseteq X$.

Proof. Let $\tilde{U} \in \tau$. Then $\tilde{U} \cap \tilde{Y}$ open in $Y$. We can choose an element $B \in \vartheta$ such that $B \subseteq \tilde{U}$. Then $B \cap \tilde{Y} \subseteq \tilde{U} \cap \tilde{Y}$. It follows from lemma 3.4 that $\vartheta_{Y}$ is a type 2 fuzzy multi base for the type 2 fuzzy multi subspace topology on $Y$.

Definition 25. Let $(X, \tau)$ be a type 2 fuzzy multi topological space. A sub collection $\zeta$ of $\tau$ is called type 2 fuzzy multi sub-base for $\tau$, if the collection of all finite type 2 fuzzy multi intersections of elements of $\zeta$ is a base for $\tau$. The type 2 fuzzy multi topology generated by the type 2 fuzzy multi sub-base $\zeta$ is defined to be the collection $\tau$ of type 2 fuzzy multi union of all finite intersections of elements of $\zeta$.

Theorem 26. Let $(X, \tau)$ be a type 2 fuzzy multi topological space and $\zeta$ be a collection of type 2 fuzzy multi sets drawn from $X$. Then $\zeta$ is a type 2 fuzzy multi sub-base for $\tau$ if and only if $\zeta$ generates $\tau$.

Proof. Let $\mathbf{B}$ be the family of finite intersections of members of $\zeta$ and $\zeta$ be a sub-base for $\tau$. It can be shown that $\tau$ is the smallest type 2 fuzzy multi topology on $X$ containing $\zeta$. Since $\zeta \subseteq \mathbf{B}$ and $\mathbf{B} \subseteq \tau, \zeta \subseteq \tau$. Suppose $\tau^{*}$ is some other type 2 fuzzy multi topology in $X$ such that $\zeta \subseteq \tau^{*}$. We have to show that $\tau \subseteq \tau^{*}$. Since $\zeta \subseteq \tau^{*}, \tau^{*}$ contains all finite intersections of members of $\zeta$, that is $\mathbf{B} \subseteq \tau^{*}$. Since $\mathbf{B}$ is a base, each member of $\tau$ can be written as the type 2 fuzzy multi union of some members of $\mathbf{B}$ and it follows that $\tau \subseteq \tau^{*}$.

Conversely suppose that $\tau$ is the smallest type 2 fuzzy multi topology on $X$ containing $\zeta$. We have to show that $\zeta$ is a sub-base for $\tau$. That is, $\mathbf{B}$ is a base for $\tau$. Suppose there is a type 2 fuzzy multi topology $\tau^{*}$ on $X$ such that $\mathbf{B}$ is a base for $\tau^{*}$. Then every member of $\tau^{*}$ can be expressed as a union of the sub family of $\mathbf{B}$ and so it is in $\tau$ since $\mathbf{B} \subseteq \tau$. This means that $\tau^{*} \subseteq \tau$ and consequently $\tau=\tau^{*}$. Since $\tau$ is the smallest topology containing $\zeta, \zeta$ is a sub-base for $\tau$. 


\section{Functions and Type 2 Fuzzy Multi Continuity}

In this section, we generalize the notion of continuity to type 2 fuzzy multi context. As a preliminary, we define functions and establish several properties of type 2 fuzzy multisets induced by functions.

Definition 27. Let $\phi$ be a mapping from $X$ into $Y$ and let $\tilde{A} \in F M_{T 2}(X)$ and $\tilde{B} \in F M_{T 2}(Y)$. The type 2 fuzzy multisets $\phi(\tilde{A}) \in F M_{T 2}(Y)$ and $\phi^{-1}(\tilde{B}) \in$ $F M_{T 2}(X)$, defined by $\forall y \in Y$,

$$
\begin{aligned}
\phi(\tilde{A})(y) & =\left\{\vee_{\phi(x)=y}\left\{\int_{i=1}^{L_{p}(x)} \sum_{j=1}^{L_{s}(x)} f_{x}^{j}\left(u_{i}\right) / u_{i}\right\}\right. & & \text { if } \phi^{-1}(y) \neq \Phi \\
& =0 & & \text { otherwise }
\end{aligned}
$$

and $\forall x \in X, \quad \phi^{-1}(\tilde{B})(x)=\tilde{B} \phi(x)$,

are called, respectively, the image of $\tilde{A}$ under $\phi$ and the pre-image (or inverse image) of $\tilde{B}$ under $\phi$.

In other words, $\phi(\tilde{A})(y)=\vee_{\phi(x)=y}\{\tilde{A}(x)\}$

$$
=0
$$

if $\phi^{-1}(y) \neq \Phi$

otherwise

and $\forall x \in X, \quad \phi^{-1}(\tilde{B})(x)=\tilde{B} \phi(x)$. In the next proposition we discuss some of the properties of function.

Proposition 28. Let $X, Y$ and $Z$ be three nonempty sets and $\phi: X \rightarrow Y$ and $\psi: Y \rightarrow Z$ be two mappings. If $A, A_{i} \in F M_{T 2}(X), B, B_{i} \in F M_{T 2}(Y)$, $C \in F M_{T 2}(Z) ; i \in I$ then
a) $\tilde{A}_{1} \subseteq \tilde{A}_{2} \Rightarrow \phi\left(\tilde{A}_{1}\right) \subseteq \phi\left(\tilde{A}_{2}\right)$
b) $\tilde{B}_{1} \subseteq \tilde{B}_{2} \Rightarrow \phi^{-1}\left(\tilde{B}_{1}\right) \subseteq \phi^{-1}\left(\tilde{B}_{2}\right)$
c) $\phi\left[\cup_{i \in I} \tilde{A}_{i}\right]=\cup_{i \in I} \phi\left[\tilde{A}_{i}\right]$
d) $\phi^{-1}\left[\cup_{i \in I} \tilde{B}_{i}\right]=\cup_{i \in I} \phi^{-1}\left[\tilde{B}_{i}\right]$
e) $\phi^{-1}\left[\cap_{i \in I} \tilde{B}_{i}\right]=\cap_{i \in I} \phi^{-1}\left[\tilde{B}_{i}\right]$
f) $\psi\left[\phi\left(\tilde{A}_{i}\right)\right]=[\psi \phi]\left(\tilde{A}_{i}\right)$ and $\phi^{-1}\left[\psi^{-1}\left(\tilde{A}_{i}\right)\right]=[\psi \phi]^{-1}\left(\tilde{A}_{i}\right)$

Proof. a) Let $\tilde{A}_{1} \subseteq \tilde{A}_{2}$.

Then $u_{i} \leq w_{i}$ for $i=1,2,3, \ldots L_{p}(x)$ and

$f_{x}^{j}\left(u_{i}\right) \leq g_{x}^{j}\left(w_{i}\right)$ for $j=1,2,3, \ldots L_{s}(x)$, for every $x \in X$

$\Rightarrow \tilde{A}_{1}(x) \leqslant \tilde{A}_{2}(x)$ for every $x \in X$

$\Rightarrow \vee_{\phi(x)=y}\left\{\tilde{A}_{1}(x)\right\} \leqslant \vee_{\phi(x)=y}\left\{\tilde{A}_{2}(x)\right\}$ for every $x \in X$

$\Rightarrow \phi\left(\tilde{A}_{1}\right)(y) \leqslant \phi\left(\tilde{A}_{2}\right)(y)$

$\Rightarrow \phi\left(\tilde{A}_{1}\right) \subseteq \phi\left(\tilde{A}_{2}\right)$

b) Let $\tilde{B}_{1} \subseteq \tilde{B}_{2}$.

Then $\tilde{B}_{1}(y) \leqslant \tilde{B}_{2}(y)$

$\Rightarrow \tilde{B}_{1} \phi(x) \leqslant \tilde{B}_{2} \phi(x)$

$\Rightarrow \phi^{-1} \tilde{B}_{1}(x) \leqslant \phi^{-1} \tilde{B}_{2}(x)$ 
$\Rightarrow \phi^{-1}\left(\tilde{B}_{1}\right) \subseteq \phi^{-1}\left(\tilde{B}_{2}\right)$.

c) Let $\tilde{A}=\cup_{i \in I} \tilde{A}_{i}$.

Then $\left.\left.\phi\left(\cup_{i \in I} \tilde{A}_{i}\right)(y)=\phi(\tilde{A})(y)=\vee_{\phi(x)=y}\{\tilde{A})\right\}(x)=\vee_{\phi(x)=y}\left\{\vee_{i \in I} \tilde{A}_{i}\right)\right\}(x)$

$$
\begin{aligned}
& \left.=\vee_{i \in I}\left\{\bigvee_{\phi(x)=y} \tilde{A}_{i}\right)\right\}(x) \\
& =\vee_{i \in I} \phi\left(\tilde{A}_{i}(y)\right) \\
& =\cup_{i \in I} \phi\left(\tilde{A}_{i}(y)\right)
\end{aligned}
$$

d)Let $\tilde{B}=\cup_{i \in I} \tilde{B}_{i}$.

$$
\phi\left[\cup_{i \in I} \tilde{A}_{i}\right]=\cup_{i \in I} \phi\left[\tilde{A}_{i}\right] .
$$

Then $\phi^{-1}\left(\cup_{i \in I} \tilde{B}_{i}\right)(x)=\phi^{-1}(\tilde{B})(x)=\tilde{B} \phi(x)=\vee_{i \in I} \tilde{B}_{i} \phi(x)=\vee_{i \in I} \phi^{-1}\left(\tilde{B}_{i}\right)(x)=$ $\cup_{i \in I} \phi^{-1}\left(\tilde{B}_{i}\right)(x)$

Hence $\phi^{-1}\left[\cup_{i \in I} \tilde{B}_{i}\right]=\cup_{i \in I} \phi^{-1}\left[\tilde{B}_{i}\right]$

e)Let $\tilde{B}=\cap_{i \in I} \tilde{B}_{i}$.

Then $\phi^{-1}\left(\cap_{i \in I} \tilde{B}_{i}\right)(x)=\phi^{-1}(\tilde{B})(x)=\tilde{B} \phi(x)=\wedge_{i \in I} \tilde{B}_{i} \phi(x)=\wedge_{i \in I} \phi^{-1}\left(\tilde{B}_{i}\right)(x)=$ $\cap_{i \in I} \phi^{-1}\left(\tilde{B}_{i}\right)(x)$

Hence $\phi^{-1}\left[\cap_{i \in I} \tilde{B}_{i}\right]=\cap_{i \in I} \phi^{-1}\left[\tilde{B}_{i}\right]$

f) $\psi[\phi(\tilde{A})](z)=\vee_{\psi(z)=y}\{\phi(\tilde{A})(y)\} ; y \in Y$

$$
\begin{aligned}
& =\vee_{\psi(z)=y}\left\{\vee_{\phi(x)=y} \phi(\tilde{A})(x)\right\} ; y \in Y, x \in X \\
& =\vee\{\vee\{\tilde{A}(x) ; x \in X, \phi(x)=y\} ; y \in Y, \psi(y)=z\} \\
& =\vee\{\tilde{A}(x) ; x \in X,[\psi \phi](x)=z\} \\
& =\vee_{[\psi \phi](x)=z}\{\tilde{A}(x) ; x \in X\} \\
& =[\psi \phi](\tilde{A})(z) .
\end{aligned}
$$

Hence $\psi[\phi(\tilde{A})]=[\psi \phi](\tilde{A})$.

Using this part and (b) we can prove the second part.

Now we are ready to define type 2 fuzzy multi continuity.

Definition 29. Let $(X, \tau)$ and $(Y, \varsigma)$ be two type 2 fuzzy multi topological spaces. A function $\phi$ from $(X, \tau)$ to $(Y, \varsigma)$ is said to be continous if and only if the inverse image of each $\varsigma$ - type 2 fuzzy multi open set is $\tau$ - type 2 fuzzy multi open set.

Clearly, if $\phi$ is a type 2 fuzzy multi continuous function from $(X, \tau)$ to $(Y, \varsigma)$ and $\psi$ is a type 2 fuzzy multi continuous function from $(Y, \varsigma)$ to $(Z, \varrho)$, then the composition $\psi \circ \phi$ is a type 2 fuzzy multi continuous function on $(X, \tau)$ to $(Z, \varrho)$, for $(\psi \circ \phi)^{-1}[\tilde{B}]=\phi^{-1}\left[\psi^{-1}[\tilde{B}]\right]$ for each type 2 fuzzy multiset $\tilde{B}$ in $Z$. ( By proposition 28(f)). Also by using the continuity of $\psi$ and $\phi$ it follows that if $\tilde{B}$ is $\varrho$ - open then $(\psi \circ \phi)^{-1}[\tilde{B}]$ is $\tau$ - open.

Next we discuss some equivalence relations of continuous functions.

Theorem 30. Let $(X, \tau)$ and $(Y, \varsigma)$ be two type 2 fuzzy multi topological spaces. A function $\phi$ from $(X, \tau)$ to $(Y, \varsigma)$ is continous if and only if the inverse 
image of each type 2 fuzzy multi closed set in $\varsigma$ is a type 2 fuzzy multi closed set in $\tau$.

Proof. Assume that $\phi$ is continuous and $\tilde{B}$ be a type 2 fuzzy multi closed set in $\varsigma$.

To prove $\phi^{-1}(\tilde{B})$ is closed, it is enough to show that $\left[\phi^{-1}(\tilde{B})\right]^{c}$ is a type 2 fuzzy multi open set in $\tau$.

For all $x \in X$, let $\tilde{B} \phi(x)=\int_{i=1}^{L_{p}(\phi(x))} \sum_{j=1}^{L_{s}(\phi(x))} f_{\phi(x)}^{j}\left(u_{i}\right) / u_{i}$. Then

$\phi^{-1}\left(\tilde{B}^{c}\right)(x)=\tilde{B}^{c} \phi(x)=\int_{i=1}^{L_{p}(\phi(x))} \sum_{j=1}^{L_{s}(\phi(x))} f_{\phi(x)}^{j}\left(u_{i}\right) /\left(1-u_{i}\right)$ and

$\left[\phi^{-1}(\tilde{B})(x)\right]^{c}=[\tilde{B} \phi(x)]^{c}=\int_{i=1}^{L_{p}(\phi(x))} \sum_{j=1}^{L_{s}(\phi(x))} f_{\phi(x)}^{j}\left(u_{i}\right) /\left(1-u_{i}\right)$.

Hence

$$
\phi^{-1}\left(\tilde{B}^{c}\right)=\left[\phi^{-1}(\tilde{B})\right]^{c}
$$

Since $\phi$ is continuous and $\tilde{B}^{c}$ is open in $\varsigma, \phi^{-1}\left(\tilde{B}^{c}\right)$ is a type 2 fuzzy multi open set in $\tau$. Therefore, $\left[\phi^{-1}(\tilde{B})\right]^{c}$ is a type 2 fuzzy multi open set in $\tau$. (by eq: 3 )

Similarly, using eq: 3 we can prove the converse part.

Theorem 31. Let $(X, \tau)$ and $(Y, \varsigma)$ be two type 2 fuzzy multi topological spaces. A function $\phi$ from $(X, \tau)$ to $(Y, \varsigma)$ is continuous if and only if for each $\tilde{A} \in F M_{T 2}(X), \phi[\operatorname{cl}(\tilde{A})] \subseteq \operatorname{cl}[\phi(\tilde{A})]$.

Proof. Assume that $\phi$ is continuous.

For any $\tilde{A} \in F M_{T 2}(X), \phi(\tilde{A}) \subseteq c l(\phi(\tilde{A}))$ (by theorem $14(2)$ )

$\tilde{A}=\phi^{-1}(\phi(\tilde{A})) \subseteq \phi^{-1}[\operatorname{cl}(\phi(\tilde{A}))]$ (by proposition $\left.28(\mathrm{~b})\right)$.

Since $\phi$ is continuous and $\operatorname{cl}[\phi(\tilde{A})]$ is closed, $\phi^{-1}[\operatorname{cl}(\phi(\tilde{A}))]$ is closed.

$\operatorname{cl}(\tilde{A}) \subseteq \phi^{-1}[\operatorname{cl}(\phi(\tilde{A}))]$ (Since $\operatorname{cl}(\tilde{A})$ is the smallest type 2 fuzzy multi closed set contains $\tilde{A})$

$\Rightarrow \phi(\operatorname{cl}(\tilde{A})) \subseteq \operatorname{cl}(\phi(\tilde{A}))$.

Conversely assume the given condition.

To prove $\phi$ is continuous, let $\tilde{B}$ be a type 2 fuzzy multi closed set in $\varsigma$.

Then by assumption, $\phi\left(\operatorname{cl}\left(\phi^{-1}(\tilde{B})\right)\right) \subseteq \operatorname{cl}\left(\phi\left(\phi^{-1}(\tilde{B})\right)\right)=\operatorname{cl}(\tilde{B})=\tilde{B}$ (by proposition $14(3))$

Thus $c l\left(\phi^{-1}(\tilde{B})\right) \subseteq \phi^{-1}(\tilde{B})$.

But $\phi^{-1}(\tilde{B}) \subseteq \operatorname{cl}\left(\phi^{-1}(\tilde{B})\right)$ (by proposition $\left.14(2)\right)$

$\Rightarrow \phi^{-1}(\tilde{B})=\operatorname{cl}\left(\phi^{-1}(\tilde{B})\right)$

$\Rightarrow \phi^{-1}(\tilde{B})$ is closed in $\tau$ ( by proposition $14(3)$ )

$\Rightarrow \phi$ is continuous. 
Theorem 32. Let $(X, \tau)$ and $(Y, \varsigma)$ be two type 2 fuzzy multi topological spaces. A function $\phi$ from $(X, \tau)$ to $(Y, \varsigma)$ is continuous if and only if for each $\tilde{B} \in F M_{T 2}(Y), \operatorname{cl}\left[\phi^{-1}(\tilde{B})\right] \subseteq \phi^{-1}[\operatorname{cl}(\tilde{B})]$.

Proof. By theorem 31, $\phi(\operatorname{cl}(\tilde{A})) \subseteq \operatorname{cl}(\phi(\tilde{A}))$. Replace $\tilde{A}$ by $\phi^{-1}(\tilde{B})$. Then $\phi\left(\operatorname{cl}\left(\phi^{-1}(\tilde{B})\right) \subseteq \operatorname{cl}\left(\phi\left(\phi^{-1}(\tilde{B})\right)\right)=\operatorname{cl}(\tilde{\tilde{B}})\right.$

$\Rightarrow \phi^{-1}\left(\phi\left(\operatorname{cl}\left(\phi^{-1}(\tilde{B})\right)\right) \subseteq \phi^{-1}(\operatorname{cl}(\tilde{B}))\right.$

$\Rightarrow \operatorname{cl}\left(\phi^{-1}(\tilde{B}) \subseteq \phi^{-1}(\operatorname{cl}(\tilde{B}))\right.$.

Conversely assume the given condition.

To prove $\phi$ is continuous, let $\tilde{B}$ be a type 2 fuzzy multi closed set in $\varsigma$.

Then by assumption $\operatorname{cl}\left(\phi^{-1}(\tilde{B}) \subseteq \phi^{-1}(\operatorname{cl}(\tilde{B}))\right.$

$\Rightarrow \operatorname{cl}\left(\phi^{-1}(\tilde{B}) \subseteq \phi^{-1}(\tilde{B})\right.$ (since $\tilde{B}$ is closed)

But $\phi^{-1}(\tilde{B}) \subseteq c l\left(\phi^{-1}(\tilde{B})\right.$ (by theorem 14(2))

Therefore, $\operatorname{cl}\left(\phi^{-1}(\tilde{B})\right)=\phi^{-1}(\tilde{B})$. Hence by theorem $14(3) \phi^{-1}(\tilde{B})$ is closed.

$\phi$ is continuous. ( by theorem 30 )

Theorem 33. Let $(X, \tau)$ and $(Y, \varsigma)$ be two type 2 fuzzy multi topological spaces. A function $\phi$ from $(X, \tau)$ to $(Y, \varsigma)$ is continuous if and only if for each $\tilde{A} \in F M_{T 2}(X),[\phi(\tilde{A})]^{\circ} \subseteq \phi\left(\tilde{A}^{o}\right)$.

Proof. Assume that $\phi$ is continuous.

For any $\tilde{A} \in F M_{T 2}(X),[\phi(\tilde{A})]^{o} \subseteq \phi(\tilde{A})$ (by theorem $12(2)$ )

$\Rightarrow \phi^{-1}\left([\phi(\tilde{A})]^{o}\right) \subseteq \tilde{A}$ (by proposition $28(\mathrm{~b})$ ) .

Since $\phi$ is continuous and $[\phi(\tilde{A})]^{o}$ is open, $\phi^{-1}\left[(\phi(\tilde{A}))^{o}\right]$ is open.

But $\tilde{A}^{o}$ is the largest type 2 fuzzy multi open set contained in $\tilde{A}$

$\Rightarrow \phi^{-1}\left(\phi(\tilde{A})^{o}\right) \subseteq \tilde{A}^{o}$

$\Rightarrow[\phi(\tilde{A})]^{o} \subseteq \phi\left(\tilde{A}^{o}\right)$

Conversely assume the given condition.

To prove $\phi$ is continuous, let $\tilde{B}$ be a type 2 fuzzy multi open set in $\varsigma$.

Then by assumption, $\left[\phi\left(\phi^{-1}(\tilde{B})\right)\right]^{o} \subseteq \phi\left(\phi^{-1}(\tilde{B})^{o}\right)$

$\Rightarrow \tilde{B}^{o} \subseteq \phi\left[\left(\phi^{-1} \tilde{B}\right)^{o}\right]$

$\Rightarrow \tilde{B} \subseteq \phi\left[\left(\phi^{-1} \tilde{B}\right)^{o}\right]$ (by theorem 12(3))

$\Rightarrow \phi^{-1}(\tilde{B}) \subseteq\left(\phi^{-1}(\tilde{B})\right)^{\circ}$

But by theorem $12(2)\left(\phi^{-1}(\tilde{B})\right)^{o} \subseteq \phi^{-1}(\tilde{B})$

$\Rightarrow \phi^{-1}(\tilde{B})=\left(\phi^{-1}(\tilde{B})\right)^{o}$

$\Rightarrow \phi^{-1}(\tilde{B})$ is open in $\tau$ (by theorem $12(3)$ )

$\Rightarrow \phi$ is continuous. 


\section{Conclusion}

Topology is an important area of pure mathematics to which fuzzy sets have been applied in a systematic and ordered manner. In this paper we focus on topology of type 2 fuzzy multisets, a combined structure of type 2 fuzzy sets and multisets. This work extends the theory of general topology to type 2 fuzzy multisets. It begins with basic definitions and operations of type 2 fuzzy multisets. The concept of type 2 fuzzy multi topological spaces are introduced. The notions and properties of open sets, closed sets, closure, interior and neighbourhoods in type 2 fuzzy multi context are introduced and investigated. Also, the concepts of base, sub-base and subspace are extended to type 2 fuzzy multi topological spaces and related theorems are proved. Further more, functions are defined and many properties are established. Finally, using these functions the concept of type 2 fuzzy multi continuity is defined and results are proved.

We hope that this work would help enhancing the study on type 2 fuzzy multi topological space to establish a general framework for practical applications.

\section{References}

[1] K. T. Attanassov, Intuitionistic fuzzy sets, Fuzzy Sets and Systems, 20, No. 1 (1986) 87-96.

[2] K. T. Attanassov, Operatiors over interval valued intuitionistic fuzzy sets, Fuzzy Sets and Systems, 64, No. 2 (1994), 159-174.

[3] W.D.Blizard, Multiset Theory, Norte Dame Journal of Formal Logic, 30, No. 1 (1989), 36-66.

[4] C.L.Chang, Fuzzy topological spaces, Journal of Mathematical Analysis and Applications, 24 (1968), 182-190.

[5] D. Coker, An introduction to intuitionistic fuzzy topological spaces, Fuzzy sets and systems, 88, No. 1 (1997), 81-89.

[6] W.L.Gau and D.J. Buehrer, Vague sets, IEEE Transactions on Systems, Man and Cybernetics, 23, No. 2 (1993), 610-614.

[7] K.P.Girish and Sunil Jacob John, Rough multisets and its multiset topology, Transactions on Rough Sets XIV (2011), 62-80.

[8] K.P.Girish and Sunil Jacob John, Multiset topologies induced by multiset relations, Information Sciences, 188 (2012), 298-313.

[9] Jaya Paul and Sunil Jacob John, Type 2 fuzzy multisets and its application in decision making problems, Journal of Intelligent Fuzzy systems, 30 (2016), 359-369.

[10] N.N.Karnik and J.M.Mendel, Operations of type 2 fuzzy sets, Fuzzy sets and systems, 122, No. 2 (2001), 327-348. 
[11] J.M.Mendel and R. I. John, Type 2 fuzzy sets made simple, IEEE Transactions on Fuzzy Systems, 10, No. 2 (2002), 117- 127.

[12] D. Molodtsov, Soft set theory- First results, Computers and Mathematics with Applications, 37, No. 4-5 (1999), 19-31.

[13] J. N.Mordeson, K. R. Bhutani and A. Rosenfeld, Fuzzy Group Theory, Springer, 182 (2005) .

[14] J. R. Munkres, Topology: a first course, Prentice Hall, 1975.

[15] Z. Pawlak, Rough sets, International Journal of Computer and Information Sciences, 11, No. 5 (1982), 341-356.

[16] S. E. Rodabaugh and E. P. Klement, Topological and algebraic structures in fuzzy sets: A handbook of recent developments in the Mathematics of fuzzy sets, 20, springer Science and Business Media, 2003.

[17] A. Rosenfeld, Fuzzy Groups, Journal of Mathematical Analysis and Applications, 35, No. 3 (1971), 512-517.

[18] T. K. Shinoj, S. J. John, Intuitionistic Fuzzy Multigroups, Annals of Pure and Applied Mathematics, 9, No. 1 (2015), 133-143.

[19] T. K. Shinoj, S. J. John, Topological Structures on Intuitionistic Fuzzy Multisets, International Journal of Scientific and Engineering Research, 6, No. 1 (2015), 192-200.

[20] G. F. Simmons, Topology and modern analysis, McGraw-Hill New York, 3, 1963.

[21] A.Syropoulos, Fuzzy Multisets and Fuzzy Computing, Handbook of Research on Generalized and Hybrid Set Structures and Applications for Soft Computing, 23, 2016.

[22] L. A. Zadeh, Fuzzy sets, Information and control, 8, No. 3 (1965), 338-353 . 
